\title{
OPTIMAL STOPPING OF THE MAXIMUM PROCESS
}

\author{
LUIS H. R. ALVAREZ ${ }^{* * *}$ AND \\ PEKKA MATOMÄKI, ${ }^{* * * *}$ University of Turku
}

\begin{abstract}
We consider a class of optimal stopping problems involving both the running maximum as well as the prevailing state of a linear diffusion. Instead of tackling the problem directly via the standard free boundary approach, we take an alternative route and present a parameterized family of standard stopping problems of the underlying diffusion. We apply this family to delineate circumstances under which the original problem admits a unique, well-defined solution. We then develop a discretized approach resulting in a numerical algorithm for solving the considered class of stopping problems. We illustrate the use of the algorithm in both a geometric Brownian motion and a mean reverting diffusion setting.
\end{abstract}

Keywords: Optimal stopping; linear diffusions; maximum process

2010 Mathematics Subject Classification: Primary 60J60; 62L15

Secondary 60G40

\section{Introduction}

Let $X_{t}$ be an Itô diffusion evolving on the state space $\mathbb{R}_{+}$, and denote by $S_{t}=\sup _{s \leq t}\left\{X_{t}\right\}$ its running supremum. In this paper our objective is to analyze and solve the infinite-horizon optimal stopping problem

$$
\sup _{\tau} \mathbb{E}_{(x, s)}\left\{\mathrm{e}^{-r \tau} f\left(X_{\tau}, S_{\tau}\right)\right\},
$$

where the exercise payoff $f(x, s)$ is assumed to be decreasing in $x$ and increasing in $s, r>0$ denotes the exogenously given constant discount rate, and $\tau$ is a stopping time. Two wellknown examples belonging to this class of stopping problems are the Russian option for which $f(x, s)=s$ (see, e.g. [19] and [27]) and the American lookback option with a floating strike for which $f(x, s)=s-x$ (see, e.g. [9] and [19]). While both of these cases constitute perpetual path-dependent options, the latter problem also has an alternative interpretation as a measure of a risk for a stock (see [10] and [20]).

Typically, optimal stopping problems of the type (1.1) are solved by considering an associated free boundary problem (for a pioneering treatment, see [25]; for a comprehensive treatment of these problems, see Chapter III and Section 13 of [26]). In [25] the considered stopping problem was of the form

$$
\sup _{\tau} \mathbb{E}_{(x, s)}\left\{F\left(S_{\tau}\right)-\int_{0}^{\tau} c\left(X_{S}\right) \mathrm{d} s\right\},
$$

Received 21 June 2012; revision received 6 August 2013.

* Postal address: Department of Accounting and Finance, Turku School of Economics, University of Turku, Turku, FI-20500, Finland.

** Email address: 1hralv@utu.fi

*** Email address: pjsila@utu.fi 
where $F$ is an increasing function, $c$ is a positive function, and $X_{t}$ is Brownian motion. In that study, a powerful maximality principle was developed. According to that principle, the first-order differential equation characterizing the optimal exercise boundary admits a maximal solution which stays strictly below the diagonal in $\mathbb{R}_{+}^{2}$. It was then shown that the maximality principle is equivalent to the existence of a finite solution, and that the optimal stopping strategy can be characterized as the first time the process $X_{t}$ falls below the maximal solution. More recently, this technique has been further refined by Obłój [22], who extended the original results of [25] to a more general setting. The optimal stopping of the running minimum within an optimal prediction of the ultimate minimum setting has recently been investigated and solved in [12] by relying on a free boundary approach. Furthermore, the maximality principle has also been adapted to problems involving spectrally negative Lévy processes (see, e.g. [18], [23], and the references therein).

In this paper we address the optimal stopping problem (1.1) under a set of reasonable basic regularity and smoothness assumptions on the exercise payoff and the underlying diffusion. Instead of relying on a free boundary approach, we take an alternative route and present a parameterized family of associated standard stopping problems which we solve explicitly by relying on ordinary optimization techniques. We subsequently apply our findings in deriving, independently of the free boundary problem, a set of sufficient conditions under which (1.1) indeed attains a finite solution. Our approach relies on the $r$-excessivity of the values of the associated stopping problems. In that way it avoids the immediate application of the smooth pasting and instantaneous reflection conditions, even though especially the former of these conditions is to some extent embedded in the considered class of optimization problems.

Having established the existence of a solution for the considered class of stopping problems, we then develop a discretized approach which can be applied to determining the optimal policy and its value. In a finite-horizon case of the problem, one can discretize time, leading to a familiar binomial tree framework similar to the well-known CRR model (see, e.g. [4] and [16]). However, within an infinite-time horizon setting this approach is no longer possible and a somewhat different discretization is required. As our study demonstrates, discretizing the state of the supremum process is an appropriate technique leading to a desired outcome. In the chosen discretization framework, the supremum process can only take values from an arithmetic sequence. Since the supremum process increases only at states where it coincides with the underlying diffusion, we note that at any given date the underlying process has hit only finitely many times its discretized supremum. Between these hitting times, the twodimensional process $\left(X_{t}, S_{t}\right)$ behaves as a one-dimensional process. It then follows that the discretized problem can be seen as a countable sequence of relatively easily solvable onedimensional subproblems. Since this sequence is shown to converge to the optimal solution under a set of typically satisfied conditions, our study complements the existing approaches by presenting a technique which does not require the analysis of the ordinary differential equation characterizing the optimal boundary. This discretization simultaneously results in an algorithm for finding the optimal threshold and value numerically as a limit of a converging sequence. In this way we do not only prove that there exists a unique threshold rule, we also identify it. For the sake of generality, we also consider an extension of the original problem (1.1) in a case where there are no monotonicity requirements for the exercise payoff $f(x, s)$. It turns out that our approach applies in that case as well, leading to a convergent sequence approaching the solution.

In order to illustrate our findings explicitly, we solve the value and optimal exercise strategy of a lookback option with a floating strike for a general Itô diffusion. We also determine the value 
and optimal stopping strategy of a $\pi$-option $\left(f(x, s)=x^{\kappa} s^{\eta}-K\right.$, with $\left.\kappa, \eta, K \geq 0\right)$ introduced in [14]. The efficiency of the developed discrete algorithm is then illustrated for these two option models under two different dynamic specifications for the underlying diffusion process. All in all, our examples seem to indicate that the discretization method can be successfully used to solve a great variety of different stopping problems involving a running supremum process, the primary restrictive factor being an $s$-Hölder continuity of $f$.

It is at this point worth mentioning that there are also other approaches that avoid the use of the free boundary conditions and the maximality principle. In [5] an alternative technique based on a measure transform was introduced. This technique, known as the Beibel-Lerche approach, has been successfully applied in the solution of some optimal stopping problems of the running supremum of a geometric Brownian motion (see [19]). Another alternative approach was developed in [15]. Instead of analyzing the free boundary problem subject to appropriate boundary conditions, Hobson [15] directly computed the expected value of stopping strategies defined with respect to a suitable class of boundaries and then chose the optimal one by relying on arguments familiar from the calculus of variations.

The contents of this study are as follows. The problem and the basic assumptions are represented in an exact form in Section 2. In Section 3 we then prove the existence of a solution to (1.1) by solving a parameterized family of associated stopping problems. We show in Section 4 that the optimal value and stopping boundary can also be found by using the discretization method. Our findings are then illustrated numerically in Sections 5 and 6.

\section{The optimal stopping problem}

Let $\left(\Omega, \mathbb{P},\left\{\mathscr{F}_{t}\right\}_{t \geq 0}, \mathcal{F}\right)$ be a complete filtered probability space satisfying the usual conditions (see [7, p. 2]). Let $X_{t}$ be a regular linear diffusion defined on $\left(\Omega, \mathbb{P},\left\{\mathcal{F}_{t}\right\}_{t \geq 0}, \mathcal{F}\right)$, evolving on $\mathbb{R}_{+}$according to the dynamics described by the Itô differential equation

$$
\mathrm{d} X_{t}=\mu\left(X_{t}\right) \mathrm{d} t+\sigma\left(X_{t}\right) \mathrm{d} W_{t}, \quad X_{0}=x .
$$

Here $W_{t}$ denotes the standard Brownian motion, and both the drift term $\mu: \mathbb{R}_{+} \rightarrow \mathbb{R}$ and volatility term $\sigma: \mathbb{R}_{+} \rightarrow \mathbb{R}_{+}$are assumed to be sufficiently smooth to guarantee the existence and uniqueness of a (weak) solution for the above stochastic differential equation (for example, if the conditions of Theorem 5.15 of [17] are met). Specifically, we assume that $\sigma(x)>0$ for $x \in \mathbb{R}_{+}$in order to avoid interior singularities. We also assume that the boundaries of the state space are natural for the process $X_{t}$. Furthermore, given the underlying diffusion $X_{t}$, we denote by

$$
S_{t}=\max \left\{s, \sup _{0<u \leq t}\left\{X_{u}\right\}\right\}, \quad S_{0}=s \geq x,
$$

the supremum up to date $t$ of the underlying diffusion. The time $t=0$ can be interpreted as the time when the considered optimal stopping problem arises, e.g. as the time when the lookback option is issued. In this light $s$ can be seen as the historical supremum of $X$, reached before the stopping problem arose, explaining the case $s>x$.

As usual, we define the differential operator associated with the underlying diffusion as

$$
\mathcal{A}=\frac{1}{2} \sigma^{2}(x) \frac{\mathrm{d}^{2}}{\mathrm{~d} x^{2}}+\mu(x) \frac{\mathrm{d}}{\mathrm{d} x},
$$

and denote by $g_{r}:=\mathcal{A}-r$ the differential operator associated with the underlying diffusion killed at the constant rate $r$. Given these differential operators, we denote by $\psi$ and 
$\varphi$ the increasing and decreasing fundamental solutions of the ordinary differential equation $\left(g_{r} u\right)(x)=0$, respectively. As is well known from the literature on linear diffusions, $B L^{\prime}(x)=$ $\psi^{\prime}(x) \varphi(x)-\varphi^{\prime}(x) \psi(x)$, where $B$ is the constant Wronskian of the fundamental solutions and

$$
L^{\prime}(x)=\exp \left(-\int^{x} \frac{2 \mu(y)}{\sigma^{2}(y)} \mathrm{d} y\right)
$$

denotes the density of the scale function of $X_{t}$. Moreover, $m^{\prime}(x)=2 /\left(\sigma^{2}(x) L^{\prime}(x)\right)$ denotes the density of the speed measure of $X_{t}$. For a complete characterization of the basic characteristics of a linear diffusion and the associated fundamental solutions, see Chapter 2 of [7].

Given the underlying diffusion and its running maximum, our objective is to analyze and solve the infinite-horizon optimal stopping problem

$$
V(x, s)=\sup _{\tau} \mathbb{E}_{(x, s)}\left\{\mathrm{e}^{-r \tau} f\left(X_{\tau}, S_{\tau}\right)\right\}
$$

under the following standing assumptions.

Assumption 2.1. We assume that the exercise payoff $f: \mathbb{R}_{+}^{2} \mapsto \mathbb{R}$ is $x$-nonincreasing, $s$ increasing, and satisfies the following conditions:

(a) $f(x, s) \in C^{2,1}\left(\mathbb{R}_{+}^{2}\right)$ for all $0<x \leq s<\infty$ and $0<f(0+, s)<\infty$ for all $s>0$;

(b) for a given $s>0$, there exists $\tilde{x}_{s} \in(0, s]$ such that $\left(g_{r} f\right)(x, s) \lesseqgtr 0$ for all $x \lesseqgtr \tilde{x}_{s}$ and that $\left(g_{r} f\right)(0+, s)<0$.

\section{Associated stopping problem}

\subsection{The auxiliary problem and its solution}

Instead of tackling the considered two-dimensional optimal stopping problem directly via variational inequalities, we now take an alternative approach and consider first an associated parameterized family of one-dimensional stopping problems of the underlying linear diffusion process. To this end, let $Q(s)$ be a (finite) nonnegative continuous function satisfying the inequality $Q(s) \geq f(s, s)$ for all $s \in \mathbb{R}_{+}$. Our first aim is to solve, for $x \leq s$, the auxiliary problem

$$
V^{Q}(x, s)=\sup _{\tau} \mathbb{E}_{x}\left\{\mathrm{e}^{-r \tau} f\left(X_{\tau}, s\right) \mathbf{1}_{\left\{\tau<\gamma_{s}\right\}}+\mathrm{e}^{-r \gamma_{s}} Q(s) \mathbf{1}_{\left\{\tau \geq \gamma_{s}\right\}}\right\},
$$

where $\gamma_{s}=\inf \left\{t \geq 0 \mid X_{t}=s\right\}$. This problem can be seen as a one-dimensional problem on the state space $(0, s]$, where the boundary $s$ is killing and, once reached, it leads to a terminal value $Q(s)$. In what follows, we will show that the set $\left\{V^{Q}\right\}$ generates a family of $r$-excessive majorants for the payoff $f$, from which we can later choose the specific $V^{Q}$ constituting the solution to the original problem (2.1). It is worth pointing out that an approach based on first exit times from open intervals has also been utilized in [13].

To attain our objective, respectively denote by

$$
\hat{\psi}(x)=\varphi(y) \psi(x)-\psi(y) \varphi(x) \quad \text { and } \quad \hat{\varphi}(x)=\varphi(x) \psi(s)-\psi(x) \varphi(s)
$$

the increasing and decreasing minimal $r$-excessive mappings for $X$ killed at the boundaries $y$ and $s, y<s$ (cf. [7, pp. 18-20]). Moreover, for the sake of notational simplicity, we also 
define, for any twice continuously differentiable $r$-harmonic function $u$ and sufficiently smooth function $g$, the functional $\left(\mathcal{L}_{u} g\right)$ as

$$
\left(\mathcal{L}_{u} g\right)(x, s)=\frac{g_{x}^{\prime}(x, s)}{L^{\prime}(x)} u(x)-\frac{u^{\prime}(x)}{L^{\prime}(x)} g(x, s) .
$$

We note that differentiating (3.2) with respect to the current state $x$ yields

$$
\left(\mathcal{L}_{u} g\right)_{x}^{\prime}(x, s)=\left(g_{r} g\right)(x, s) u(x) m^{\prime}(x),
$$

owing to the assumed $r$-harmonicity of the function $u(x)$.

We now restrict our analysis to ordinary first passage time type stopping rules $\tau_{y}=\inf \{t \geq$ $\left.0 \mid X_{t} \leq y\right\}$ and consider, for a given upper boundary $s \in \mathbb{R}_{+}$and initial state $x \in[y, s]$ the functional

$$
\begin{aligned}
v(y, x, s) & =\mathbb{E}_{(x, s)}\left\{\mathrm{e}^{-r \tau_{y}} f\left(X_{\tau_{y}}, s\right) \mathbf{1}_{\left\{\tau_{y}<\gamma_{s}\right\}}+\mathrm{e}^{-r \gamma_{s}} Q(s) \mathbf{1}_{\left\{\tau_{y} \geq \gamma_{s}\right\}}\right\} \\
& =\frac{\hat{\varphi}(x)}{\hat{\varphi}(y)} f(y, s)+\frac{\hat{\psi}(x)}{\hat{\psi}(s)} Q(s) .
\end{aligned}
$$

Having stated the associated valuation (3.3), we will show that there exists a unique threshold $a_{s}^{Q}=a(s, Q) \in(0, s]$ maximizing the functional $v(y, x, s)$ as a function of the boundary $y$. Moreover, we will prove that the associated stopping rule $\tau_{a_{s} Q}$ constitutes the optimal stopping rule for the auxiliary problem (3.1). We first observe by differentiating the functional $v(y, x, s)$ with respect to $y$ that

$$
\frac{\partial v(y, x, s)}{\partial y}=\frac{\hat{\varphi}(x) L^{\prime}(y)}{\hat{\varphi}^{2}(y)}\left\{\left(\mathcal{L}_{\hat{\varphi}} f\right)(y, s)-B Q(s)\right\} .
$$

Consequently, we find that a maximizing threshold exists provided that the difference in the brackets changes sign from positive to negative only once on the state space $(0, s]$. This result is established in the following auxiliary lemma.

Lemma 3.1. There exists a unique maximizing threshold $a_{s}^{Q} \in\left(0, \tilde{x}_{s}\right]$ satisfying the ordinary first-order condition $\left(\mathcal{L}_{\hat{\varphi}} f\right)\left(a_{s}^{Q}, s\right)=B Q(s)$.

Proof. Consider the functional $H(x, s)=\left(\mathcal{L}_{\hat{\varphi}} f\right)(x, s)-B Q(s)$. We first note that $\lim _{x \uparrow s}$ $H(x, s) \leq 0$, demonstrating that $H(x, s)$ is nonpositive at the upper boundary $s$. On the other hand, since $H_{x}^{\prime}(x, s)=\left(g_{r} f\right)(x, s) \hat{\varphi}(x) m^{\prime}(x)$, the functional $H(x, s)$ can be re-expressed as

$$
H(x, s)=B(f(s, s)-Q(s))-\int_{x}^{s}\left(g_{r} f\right)(t, s) \hat{\varphi}(t) m^{\prime}(t) \mathrm{d} t,
$$

showing that $H(x, s)<0$ for all $x \in\left(\tilde{x}_{s}, s\right]$ by Assumption 2.1(b). Moreover, for $x<x_{1}<\tilde{x}_{s}$, applying the mean value theorem for integrals, we obtain

$$
\begin{aligned}
H(x, s) & =H\left(x_{1}, s\right)-\int_{x}^{x_{1}}\left(g_{r} f\right)(t, s) \hat{\varphi}(t) m^{\prime}(t) \mathrm{d} t \\
& =H\left(x_{1}, s\right)-\frac{1}{r}\left(g_{r} f\right)(\xi, s)\left\{\frac{\hat{\varphi}^{\prime}\left(x_{1}\right)}{L^{\prime}\left(x_{1}\right)}-\frac{\hat{\varphi}^{\prime}(x)}{L^{\prime}(x)}\right\},
\end{aligned}
$$

where $\xi \in\left(x, x_{1}\right)$. The assumed boundary behavior together with Assumption 2.1(b) guarantees that $H(0+, s)=\infty$. Combining this observation with the continuity and monotonicity of the functional $H(x, s)$ then completes the proof of the existence and uniqueness of the maximizing boundary $a_{s}^{Q} \in\left(0, \tilde{x}_{s}\right)$. 
Having demonstrated that there is a unique boundary maximizing the functional (3.3), we are now in position to prove the following result.

Theorem 3.1. Let Assumption 2.1 hold. Then, for a given s, $\tau_{a_{s}^{Q}}=\inf \left\{t \geq 0 \mid X_{t} \leq a_{s}^{Q}\right\}$ is the optimal stopping time for the problem (3.1) and the value is

$$
V^{Q}(x, s)= \begin{cases}v\left(a_{s}^{Q}, x, s\right), & x \in\left(a_{s}^{Q}, s\right], \\ f(x, s), & x \in\left(0, a_{s}^{Q}\right] .\end{cases}
$$

Moreover, if $Q(s)$ is differentiable then

$$
\lim _{x \uparrow s} \frac{\partial V^{Q}(x, s)}{\partial s}=\left\{Q^{\prime}(s) B-\left(\mathcal{L}_{\Phi} f\right)\left(a_{s}^{Q}, s\right)\right\} \frac{\hat{\varphi}^{2}\left(a_{s}^{Q}\right)}{B},
$$

where $\Phi(x)=\varphi(x) \psi^{\prime}(s)-\varphi^{\prime}(s) \psi(x)$ denotes the minimal decreasing $r$-excessive function for the underlying diffusion reflected at $s$.

Proof. Let $V^{Q}(x, s)$ be the solution to (3.1), and denote by $J(x, s)$ the value given in (3.4). Obviously, $J(x, s)$ is obtained by following an admissible stopping strategy and, therefore, $V^{Q}(x, s) \geq J(x, s)$. In order to prove the opposite inequality, we first observe that it is clear by construction that $J(x, s)$ is continuous on $(0, s]$ and that $J_{x}^{\prime}\left(a_{s}^{Q}-, s\right)=f_{x}^{\prime}\left(a_{s}^{Q}, s\right)$. Furthermore, since

$$
\frac{\partial v\left(a_{s}^{Q}, x, s\right)}{\partial x}=\frac{\varphi\left(a_{s}^{Q}\right) Q(s)-\varphi(s) f\left(a_{s}^{Q}, s\right)}{\hat{\varphi}\left(a_{s}^{Q}\right)} \psi^{\prime}(x)+\frac{\psi(s) f\left(a_{s}^{Q}, s\right)-\psi\left(a_{s}^{Q}\right) Q(s)}{\hat{\varphi}\left(a_{s}^{Q}\right)} \varphi^{\prime}(x),
$$

we find, by letting $x \downarrow a_{s}^{Q}$ and invoking the optimality condition $\left(\mathcal{L}_{\hat{\varphi}} f\right)\left(a_{s}^{Q}, s\right)=B Q(s)$, that $J_{x}^{\prime}\left(a_{s}^{Q}+, s\right)=f_{x}^{\prime}\left(a_{s}^{Q}, s\right)$, proving the continuous differentiability of $J(x, s)$. Next, let $x \in\left(a_{s}^{Q}, s\right]$. Then $v\left(a_{s}^{Q}, x, s\right) \geq v(x-, x, s)=f(x, s)$, where the inequality follows from the optimality of $a_{s}^{Q}$. This shows that $J(x, s)$ is a continuously differentiable majorant of $f(x, s)$.

It remains to establish that $J(x, s)$ is $r$-excessive for the underlying diffusion $X$ killed at $s$. To see that this is indeed the case, we first observe that $\left(g_{r} J\right)(x, s)=0$ on $\left(a_{s}^{Q}, s\right]$ and $\left(g_{r} J\right)(x, s)=\left(g_{r} f\right)(x, s)<0$ on $\left(0, a_{s}^{Q}\right)$. The alleged result then follows from the inequality $\left|f_{x x}^{\prime \prime}\left(a_{s}^{Q} \pm, s\right)\right|<\infty$. We have thus established that $J(x, s)$ is an $r$-excessive majorant of $f(x, s)$. Since the optimal value $V^{Q}$ is the smallest of such majorants, we conclude that $J \geq V^{Q}$.

Finally, differentiating the value $J(x, s)$ with respect to $s$ and invoking the optimality condition $\left(\mathcal{L}_{\hat{\varphi}} f\right)\left(a_{s}^{Q}, s\right)=Q(s) B$ then yields (3.5).

Given the assumed differentiability of the exercise payoff, we note by implicit differentiation that the sensitivity of the optimal threshold with respect to changes in the exogenous upper boundary $s$ can be expressed as

$$
a_{s}^{Q^{\prime}}=\frac{B Q^{\prime}(s)-\left(\mathcal{L}_{\Phi} f\right)\left(a_{s}^{Q}, s\right)-\left(\mathcal{L}_{\hat{\varphi}} f_{s}\right)\left(a_{s}^{Q}, s\right)}{\left(g_{r} f\right)\left(a_{s}^{Q}, s\right) \hat{\varphi}\left(a_{s}^{Q}\right) m^{\prime}\left(a_{s}^{Q}\right)} .
$$

On the other hand, Theorem 3.1 guarantees that $V^{Q}(x, s)$ constitutes an excessive majorant of the exercise payoff as long as the inequality $Q(s) \geq f(s, s)$ is fulfilled. Combining these observations shows that if $Q(s)$ is chosen so that the condition $\left(\mathcal{L}_{\Phi} f\right)\left(a_{s}^{Q}, s\right)=B Q^{\prime}(s)$ is also satisfied, then the value $V^{Q}(x, s)$ satisfies the instantaneous reflection condition $V_{s}^{Q^{\prime}}(s-, s)=0$ 
as well and the optimal exercise boundary satisfies the differential equation

$$
a_{s}^{Q^{\prime}}=\frac{1}{2} \sigma^{2}\left(a_{s}^{Q}\right) \frac{\hat{\varphi}^{\prime}\left(a_{s}^{Q}\right) f_{s}^{\prime}\left(a_{s}^{Q}, s\right)-\hat{\varphi}\left(a_{s}^{Q}\right) f_{x s}^{\prime \prime}\left(a_{s}^{Q}, s\right)}{\left(g_{r} f\right)\left(a_{s}^{Q}, s\right) \hat{\varphi}\left(a_{s}^{Q}\right)} .
$$

It is worth pointing out that utilizing the standard free boundary approach for solving the considered stopping problem results in the differential equation (3.6) as well (cf. Section 13 of [26]).

\subsection{The solution to the main problem}

Before proving our main existence theorem for (2.1), we first need to ensure the finiteness of the value of the stopping problem.

Lemma 3.2. Let Assumption 2.1 hold, and assume that $\int_{0}^{\infty} \mathbb{E}_{(x, s)}\left\{\mathrm{e}^{-r t} f\left(0, S_{t}\right)\right\} \mathrm{d} t<\infty$ for all $0<x \leq s<\infty$. Then the value function (2.1) is finite.

Proof. Fix $0<x \leq s<\infty$, and denote by $T_{r} \sim \operatorname{Exp}(r)$ an exponentially distributed random time, independent of $W_{t}$. Since $f$ is continuous and $\left(X_{t}, S_{t}\right)$ is a strong Markov process, it is known (see, e.g. Proposition 2.1 of [11] and also Lemma 2.2 of [8]) that

$$
u(x, s):=\mathbb{E}_{(x, s)}\left\{\sup _{0 \leq t \leq T_{r}} f\left(0, S_{t}\right)\right\}=\mathbb{E}_{(x, s)}\left\{f\left(0, S_{T_{r}}\right)\right\}
$$

is $r$-excessive. Moreover, it is clear that

$$
f(x, s) \leq f(0, s)=\mathbb{E}_{(x, s)}\{f(0, s)\} \leq \mathbb{E}_{(x, s)}\left\{f\left(0, S_{T_{r}}\right)\right\}=u(x, s),
$$

demonstrating that $u$ dominates $f$. Since $V$ constitutes the minimal r-excessive majorant of $f$, we note that $V \leq u$.

Furthermore, by straightforward calculations,

$$
u(x, s)=\mathbb{E}_{(x, s)}\left\{f\left(0, S_{T_{r}}\right)\right\}=\mathbb{E}_{(x, s)}\left\{r \int_{0}^{\infty} \mathrm{e}^{-r t} f\left(0, S_{t}\right) \mathrm{d} t\right\}=r \int_{0}^{\infty} \mathbb{E}_{(x, s)}\left\{\mathrm{e}^{-r t} f\left(0, S_{t}\right)\right\} \mathrm{d} t .
$$

The last term on the right-hand side of this equality is finite by assumption and, thus, $V(x, s) \leq$ $u(x, s)<\infty$.

Having established the finiteness of the value of the optimal stopping strategy we are now in position to state our main theorem characterizing the value and optimal exercise policy of problem (2.1).

Theorem 3.2. Let Assumption 2.1 hold, and assume that $\int_{0}^{\infty} \mathbb{E}_{(x, s)}\left\{\mathrm{e}^{-r t} f\left(0, S_{t}\right)\right\} \mathrm{d} t<\infty$. Then there exists a unique function $a_{s}^{*} \in\left(0, \tilde{x}_{s}\right)$ such that $\tau_{a_{s}^{*}}=\inf \left\{t \geq 0 \mid X_{t} \leq a_{S_{t}}^{*}\right\}$ is the optimal stopping time for the considered problem (2.1). Moreover, there exists a unique $Q(s)$ for which the value $V(x, s)$ reads as in (3.4).

Proof. For $x \leq s$, problem (2.1) can be rewritten as

$$
\sup _{\tau} \mathbb{E}_{(x, s)}\left\{\mathrm{e}^{-r \tau} f\left(X_{\tau}, s\right) \mathbf{1}_{\left\{\tau<\gamma_{s}\right\}}+\mathrm{e}^{-r \gamma_{s}} \sup _{\xi} \mathbb{E}_{\{s, s\}}\left\{\mathrm{e}^{-r \xi} f\left(X_{\xi}, S_{\xi}\right)\right\} \mathbf{1}_{\left\{\tau \geq \gamma_{s}\right\}}\right\},
$$

where $\gamma_{s}=\inf \left\{t \geq 0 \mid X_{t}=s\right\}$, so that it is of the form of the auxiliary problem (3.1), with

$$
Q(s)=\sup _{\xi} \mathbb{E}_{\{s, s\}}\left\{\mathrm{e}^{-r \xi} f\left(X_{\xi}, S_{\xi}\right)\right\} .
$$


Obviously, $Q(s) \geq f(s, s)$ and, by Lemma 3.2, $Q(s)<\infty$ for all $s<\infty$. Therefore, the claim now follows from Theorem 3.1.

Theorem 3.2 states a set of sufficient conditions under which the auxiliary stopping problem constitutes the value of the optimal stopping problem (2.1). The sensitivity of the value and optimal boundary with respect to changes in the volatility of the underlying diffusion are now summarized in the following result.

Theorem 3.3. Assume that the conditions of Theorem 3.2 are satisfied, that the difference $\mu(x)-r x$ is nonincreasing, and that a transversality condition $\lim _{t \rightarrow \infty} \mathbb{E}_{x}\left\{\mathrm{e}^{-r t} X_{t}\right\}=0$ holds. Then the value function is strictly convex as a function of the current state $x$ on the continuation set $\left(a_{s}^{*}, s\right]$, and increased volatility increases the value $V(x, s)$ and decreases the optimal stopping boundary $a_{s}^{*}$.

Proof. Fix $s<\infty$, and let $\sigma_{1}(x) \leq \sigma_{2}(x)$ for all $x$. For $i=1,2$, denote by $V_{i}$ and $a_{i}^{*}$ the value function and the optimal stopping boundary for the problem (2.1), respectively, with respect to $\sigma_{i}$. The assumed monotonicity of the difference $\mu(x)-r x$ together with the transversality condition guarantee that the fundamental solutions are convex (see Corollary 1 of [2]). Furthermore, the $r$-excessivity of the value $V(x, s)$ implies that it constitutes a positive affine transformation of the minimal solutions $\psi(x)$ and $\varphi(x)$ on the set $\left(a_{s}^{*}, s\right)$ where it is $r$-harmonic, and, consequently, it is convex there. Since the sign of the relationship between increased volatility and the value of an $r$-excessive mapping is positive on the set where it is $r$-harmonic (cf. Theorem 4 of [2]), we find that $V_{1}(x, s) \leq V_{2}(x, s)$. Suppose, contrary to our claim, that $a_{1}^{*}<a_{2}^{*}$, and let $x \in\left(a_{1}^{*}, a_{2}^{*}\right)$ so that $x$ is in the continuation region with respect to $V_{1}$, and in the stopping region with respect to $V_{2}$. Then $V_{2}(x, s)=f(x, s)<V_{1}(x, s)$, which contradicts the inequality derived above.

Theorem 3.3 states a set of conditions under which increased volatility unambiguously increases the value of the optimal stopping policy and postpones exercise by lowering the optimal boundary.

\subsection{A useful extension}

It turns out that our existence result can be directly generalized to also cover a general class of continuous exercise payoffs satisfying a boundedness condition. To this end, we consider the problem (2.1) under the following weakened assumptions.

Assumption 3.1. For each $s>0$, let $\underline{x}_{s} \in[0, s]$ be the point at which $f(x, s)$ is maximized. Assume also that the exercise payoff $f: \mathbb{R}_{+}^{2} \mapsto \mathbb{R}$ satisfies the following conditions.

(a) $f(x, s) \in C\left(\mathbb{R}_{+}^{2}\right)$ for all $0<x \leq s<\infty$ and $0<f\left(\underline{x}_{s}, s\right)<\infty$ for all $s>0$.

(b) $\mathbb{E}_{(x, s)}\left\{\sup _{0 \leq t \leq T_{r}} f\left(\underline{x}_{S_{t}}, S_{t}\right)\right\}<\infty$.

Under this assumption, we can again constitute the auxiliary problem (3.1) for a nonnegative, continuous $Q(s)$ satisfying $Q(s) \geq f(s, s)$, and the following proposition holds. (Denote by $\tau_{Q}^{*}$ and $\tau^{*}$ the optimal stopping times for auxiliary problem (3.1) and (2.1), respectively.)

Proposition 3.1. Let Assumption 3.1 hold. Then

(a) the value $V(x, s)$ is finite;

(b) there exists a unique $Q(s)$ such that $V^{Q}(x, s)=V(x, s)$. Moreover, if $\tau_{Q}^{*}<\infty$ a.s., $\tau^{*}=\tau_{Q}^{*}$. 
Proof. (a) The proof is completely analogous to that of Lemma 3.2.

(b) For each finite $Q(s)$, the linear auxiliary problem (3.1) has a solution by general existence results concerning linear diffusions (see, e.g. [26]). Establishing the alleged claim is analogous to the proof of Theorem 3.2.

Interestingly, the unique existence of a solution to the general problem involving a maximum process can be reduced to the search for a unique solution to a linear diffusion problem. However, with general assumptions we cannot, naturally, guarantee the shape of the stopping region. The following corollary presents an example of how this existence result can be used (cf. Proposition 6.1 below).

Corollary 3.1. Let Assumption 3.1 hold. Furthermore, assume that, for all $s>0$ and $Q(s)$, there exists a unique stopping region $\mathfrak{S}_{s}^{Q}$ such that $\tau_{Q}^{*}=\left\{t \geq 0 \mid X_{t} \in \mathfrak{S}_{s}^{Q}\right\}$ is the optimal stopping time for the auxiliary problem (3.1). Then, for each $s>0$, there exists a unique stopping region $\mathfrak{S}_{s} \subset[0, s]$ such that $\tau^{*}=\left\{t \geq 0 \mid X_{t} \in \mathfrak{S}_{S_{t}}\right\}$, where $\mathfrak{S}_{s}=\mathfrak{S}_{S}^{Q}$ for some $Q$.

\section{The discretization}

Our objective is now to develop a sequence of optimal stopping problems by discretizing the state of the supremum process and to show that the sequence converges in the limit to the original stopping problem (2.1). To this end, we need the following two additional assumptions.

Assumption 4.1. Assume, in addition to Assumption 2.1, that

(a) $\int_{0}^{\infty} \mathbb{E}_{(x, s)}\left\{\mathrm{e}^{-r t} f\left(0, S_{t}\right)\right\} \mathrm{d} t<\infty$ for all $0<x \leq s<\infty$;

(b) $f(x, s)$ is $s$-Hölder continuous, i.e. there exist $M>0$ and $0<\alpha \leq 1$ such that $\left|f\left(x, s_{1}\right)-f\left(x, s_{2}\right)\right| \leq M\left|s_{1}-s_{2}\right|^{\alpha}$ for all $s_{1}, s_{2} \in \mathbb{R}_{+}$and $x<\min \left\{s_{1}, s_{2}\right\}$.

First of all, let us prove an equivalent condition to Assumption 4.1(a).

Lemma 4.1. Let Assumption 2.1 hold. Then $\int_{s}^{\infty}\left(f_{s}^{\prime}(0, u) / \psi(u)\right) \mathrm{d} u<\infty$ for all $s \in \mathbb{R}_{+}$if and only if Assumption 4.1(a) holds.

Proof. Let $T \sim \operatorname{Exp}(r)$. From the proof of Lemma 3.2 we know that $\mathbb{E}_{(x, s)}\left\{f\left(0, S_{T}\right)\right\}=$ $r \int_{0}^{\infty} \mathbb{E}_{(x, s)}\left\{\mathrm{e}^{-r t} f\left(0, S_{t}\right)\right\} \mathrm{d} t$.

Observe that (see [7, p. 26]), for all $x<y$, we have $\mathbb{P}_{x}\left(S_{T} \leq y\right)=\mathbb{P}_{x}\left(\tau_{y}>T\right)=$ $1-\psi(x) / \psi(y)$, where $\tau_{y}=\inf \left\{t \geq 0 \mid X_{t} \geq y\right\}$. Using this fact and Fubini's theorem, we can calculate

$$
\begin{aligned}
r \int_{0}^{\infty} \mathbb{E}_{(x, s)}\left\{\mathrm{e}^{-r t} f\left(0, S_{t}\right)\right\} \mathrm{d} t & =\mathbb{E}_{(x, s)}\left\{f\left(0, S_{T}\right)\right\} \\
& =\int_{s}^{\infty} f(0, y) \mathrm{d} \mathbb{P}\left(S_{T} \leq y\right) \\
& =\int_{s}^{\infty} f(0, y) \frac{\psi(x) \psi^{\prime}(y)}{\psi^{2}(y)} \mathrm{d} y \\
& =f(0, s)+\psi(x) \int_{s}^{\infty} \frac{f_{s}^{\prime}(0, u)}{\psi(u)} \mathrm{d} u,
\end{aligned}
$$

whence the claim follows. 


\subsection{Introducing the recursive algorithm}

Fix a step size $z>0$, number of steps $n \in \mathbb{N}$, and starting points $\left(X_{0}, S_{0}\right)=(x, s)$ with $0<x<s$, and, for all $s$, fix a terminal payoff function $Q(s)$, which satisfies $Q(s) \geq f(s, s)$ and $\lim _{t \rightarrow \infty} \mathbb{E}_{(x, s)}\left\{\mathrm{e}^{-r t} Q\left(S_{t}\right)\right\}=0$. Define $s_{k}:=s+k z$ for $k \in \mathbb{N}$. From now on in this section we assume that the supremum process $S_{t}$ can only take values $s_{k}, k=0,1,2, \ldots, n$ (for convenience, we define $\left.s:=s_{0}\right)$. That is, as we start from $(x, s)$, the supremum process $S_{t}$ jumps to the state $s_{1}$ when the diffusion $X_{t}$ reaches the point $s$ and we 'restart' $\left(X_{t}, S_{t}\right)$ from the state $\left(s, s_{1}\right)$. Again, when $X_{t}$ reaches the new supremum value $s_{1}$, the process $S_{t}$ jumps to $s_{2}$ and we again 'restart' $\left(X_{t}, S_{t}\right)$, now from $\left(s_{1}, s_{2}\right)$. The discretization is graphically illustrated in Figure 1. It is worth mentioning that, since $S_{t}$ takes values from a finite arithmetic sequence, we know that at any time $t>0$ there has been only finitely many jumps in the path of the discretized supremum. Furthermore, we consider $s_{n}$ to be the highest possible level for $X_{t}$, and, consequently, for $S_{t}$. This means that, when $X_{t}$ reaches $s_{n}$, the process is stopped (killed) and we receive the terminal payoff $Q\left(s_{n}\right)$ at that state.

Having presented the discretized version of the running supremum of the underlying diffusion, we now apply the findings of our Theorem 3.1 and define recursively a sequence of continuously differentiable $r$-excessive values dominating the exercise payoff. To this end, we first define the terminal value of the sequence as $V_{n+1} \equiv Q\left(s_{n}\right)$. Given the terminal value $V_{n+1}$, we now define recursively for any index $1 \leq k \leq n$ the values $V_{k}$ as $V_{k}:=J\left(s_{k-1}, s_{k}\right)$,

$$
\begin{aligned}
J\left(x, s_{k}\right)= & \sup _{\tau} \mathbb{E}_{\left(x, s_{k}\right)}\left\{\mathrm{e}^{-r \tau} f\left(X_{\tau}, s_{k}\right) \mathbf{1}_{\left\{\tau<\gamma_{s_{k}}\right\}}+\mathrm{e}^{-r \gamma_{s_{k}}} V_{k+1} \mathbf{1}_{\left\{\tau \geq \gamma_{s_{k}}\right\}}\right\} \\
= & \begin{cases}\frac{\varphi(x) \psi\left(s_{k}\right)-\psi(x) \varphi\left(s_{k}\right)}{\varphi\left(\hat{a}_{s_{k}}\right) \psi\left(s_{k}\right)-\psi\left(\hat{a}_{s_{k}}\right) \varphi\left(s_{k}\right)} f\left(\hat{a}_{s_{k}}, s_{k}\right) \\
+\frac{\psi(x) \varphi\left(\hat{a}_{s_{k}}\right)-\varphi(x) \psi\left(\hat{a}_{s_{k}}\right)}{\psi\left(s_{k}\right) \varphi\left(\hat{a}_{s_{k}}\right)-\varphi\left(s_{k}\right) \psi\left(\hat{a}_{s_{k}}\right)} V_{k+1}, & x \in\left(\hat{a}_{s_{k}}, s_{k}\right], \\
f\left(x, s_{k}\right), & x \in\left(0, \hat{a}_{s_{k}}\right] .\end{cases}
\end{aligned}
$$

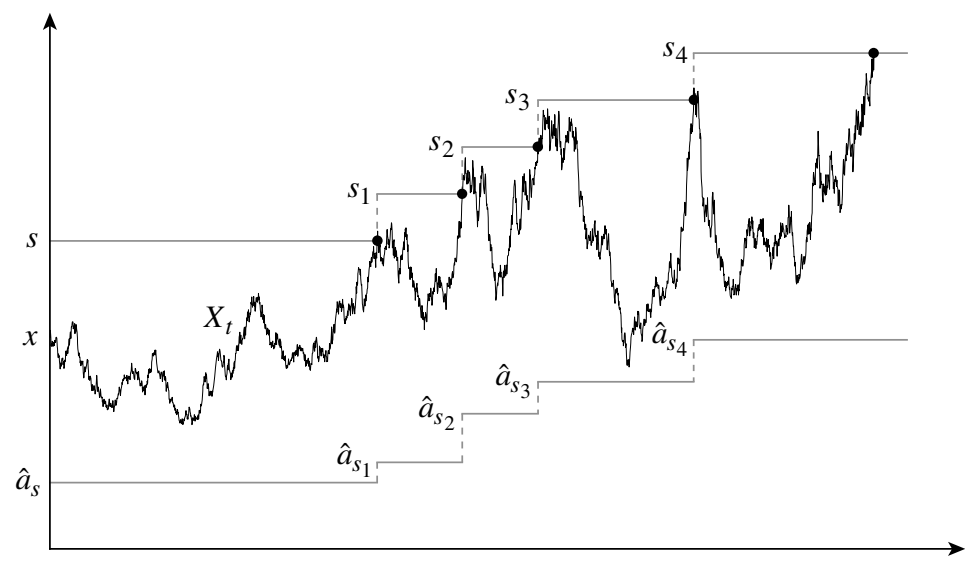

Figure 1: An illustrative example of how a sample path evolves in the constructed discretized problem. Here $n=4$ and $\hat{a}_{s_{k}}$ is the optimal stopping boundary at step $k$ for $k=0,1,2,3,4$. We stop immediately after the diffusion hits either the lower boundary $\hat{a}_{s_{k}}$, when we receive a payoff $f\left(\hat{a}_{k}, s_{k}\right)$, or the maximum level $s_{4}$, implying payoff $Q\left(s_{4}\right)$ (exogenously given terminal function). 
Here $\gamma_{s_{k}}=\inf \left\{t \geq 0 \mid X_{t}=s_{k}\right\}$ for $k=1, \ldots, n$ denotes the first hitting time of $X$ to the state $s_{k}$, and $\hat{a}_{s_{k}} \in\left(0, \tilde{x}_{s_{k}}\right)$ constitutes the unique root of the ordinary first-order condition $\left(\mathcal{L}_{\hat{\varphi}} f\right)\left(\hat{a}_{s_{k}}, s_{k}\right)=B V_{k+1}$ (cf. Theorem 3.1). Finally, the initial value is chosen as $V_{0}=J(x, s)$. It is clear that these identities completely characterize the sequence of values $\left\{V_{k}\right\}_{k=0}^{n+1}$ and the sequence of optimal exercise boundaries $\left\{\hat{a}_{s_{k}}\right\}_{k=0}^{n}$. Moreover, we also observe that, for $z>0$ and $n \in \mathbb{N}$, this discretized problem can be written in the compact form

$$
\begin{aligned}
J(z, n, x, s) & :=\sup _{\tau} \mathbb{E}_{(x, s)}\left\{\mathrm{e}^{-r \tau} f\left(X_{\tau}, s+z \sum_{k=1}^{n-1} \mathbf{1}_{\left\{\gamma_{s_{k}}<\tau\right\}}\right) \mathbf{1}_{\left\{\tau<\gamma_{s_{n}}\right\}}+\mathrm{e}^{-r \gamma_{s_{n}}} Q\left(s_{n}\right) \mathbf{1}_{\left\{\tau \geq \gamma_{s_{n}}\right\}}\right\} \\
& =\sup _{\tau} \mathbb{E}_{(x, s)}\left\{\mathrm{e}^{-r \tau} f\left(X_{\tau}, S_{\tau}\right) \mathbf{1}_{\left\{\tau<\gamma_{s_{n}}\right\}}+\mathrm{e}^{-r \gamma_{s_{n}}} Q\left(s_{n}\right) \mathbf{1}_{\left\{\tau \geq \gamma_{s_{n}}\right\}}\right\}
\end{aligned}
$$

where $S_{t}$ denotes the discretized supremum process.

In this section we shall denote by $S_{t}$ a discretized supremum process, whereas the normal continuous process is denoted by $S_{t}^{0}$.

\subsection{Proving that the algorithm works}

Let us first establish that the limiting value function of the sequence does not depend on the choice of terminal value function $Q(s)$.

Lemma 4.2. Let Assumption 4.1 hold, and fix $s, z>0$. Furthermore, let $Q(s) \geq f(s, s)$ be such that $\lim _{t \rightarrow \infty} \mathbb{E}_{(x, s)}\left\{\mathrm{e}^{-r t} Q\left(S_{t}^{0}\right)\right\}=0$. Then the limit $\lim _{n \rightarrow \infty} J(z, n, x, s)$ does not depend on the choice of $Q(s)$.

Proof. Fix $n \in \mathbb{N}$ and $Q_{1}(s)>Q_{2}(s)$. For $i=1,2$, denote by $J^{i}$ the value function associated to the terminal payoff $Q_{i}(s)$, and let $\tau_{1}$ be the optimal stopping rule maximizing the discretized problem with $Q_{1}(s)$ as a terminal value (this exists by Theorem 3.1). Since $Q_{1}(s)>Q_{2}(s)$, we know that $J^{1}(z, n, x, s)-J^{2}(z, n, x, s) \geq 0$. On the other hand, we can apply (4.2) to make an estimate:

$$
\begin{aligned}
& J^{1}(z, n, x, s)-J^{2}(z, n, x, s) \\
&= \mathbb{E}_{(x, s)}\left\{\mathrm{e}^{-r \tau_{1}} f\left(X_{\tau_{1}}, S_{\tau_{1}}\right) \mathbf{1}_{\left\{\tau_{1}<\gamma_{s_{n}}\right\}}+\mathrm{e}^{-r \gamma_{s_{n}}} Q_{1}\left(s_{n}\right) \mathbf{1}_{\left\{\tau_{1} \geq \gamma_{s_{n}}\right\}}\right\} \\
& \quad-\sup _{\tau} \mathbb{E}_{(x, s)}\left\{\mathrm{e}^{-r \tau} f\left(X_{\tau}, S_{\tau}\right) \mathbf{1}_{\left\{\tau<\gamma_{s_{n}}\right\}}+\mathrm{e}^{-r \gamma_{s_{n}}} Q_{2}\left(s_{n}\right) \mathbf{1}_{\left\{\tau \geq \gamma_{s_{n}}\right\}}\right\} \\
& \leq \mathbb{E}_{(x, s)}\left\{\mathrm{e}^{-r \tau_{1}} f\left(X_{\tau_{1}}, S_{\tau_{1}}\right) \mathbf{1}_{\left\{\tau_{1}<\gamma_{s_{n}}\right\}}+\mathrm{e}^{-r \gamma_{s_{n}}} Q_{1}\left(s_{n}\right) \mathbf{1}_{\left\{\tau_{1} \geq \gamma_{s_{n}}\right\}}\right\} \\
&-\mathbb{E}_{(x, s)}\left\{\mathrm{e}^{-r \tau_{1}} f\left(X_{\tau_{1}}, S_{\tau_{1}}\right) \mathbf{1}_{\left\{\tau_{1}<\gamma_{s_{n}}\right\}}+\mathrm{e}^{-r \gamma_{s_{n}}} Q_{2}\left(s_{n}\right) \mathbf{1}_{\left\{\tau_{1} \geq \gamma_{s_{n}}\right\}}\right\} \\
&= \mathbb{E}_{(x, s)}\left\{\mathrm{e}^{-r \gamma_{s_{n}}}\left(Q_{1}\left(s_{n}\right)-Q_{2}\left(s_{n}\right)\right) \mathbf{1}_{\left\{\tau_{1} \geq \gamma_{s_{n}}\right\}}\right\} \\
& \leq \mathbb{E}_{(x, s)}\left\{\mathrm{e}^{-r \gamma_{s_{n}}}\left(Q_{1}\left(s_{n}\right)-Q_{2}\left(s_{n}\right)\right)\right\} .
\end{aligned}
$$

Since $\lim _{n \rightarrow \infty} \mathbb{E}_{(x, s)}\left\{\mathrm{e}^{-r \gamma_{s_{n}}} Q_{i}\left(s_{n}\right)\right\}=0$ for $i=1,2$, by assumption, we note that the last term tends to 0 as $n$ approaches $\infty$.

According to Lemma 4.2 the algorithm results into the same value irrespective of the chosen terminal value $Q(s)$ as long as it satisfies the relatively weak conditions of our lemma. Hence, depending on the precise form of the exercise payoff and its behavior at the upper boundary $s$, natural choices for $Q(s)$ are, for example, additive forms $Q(s)=f(s, s)+a, a \geq 0$, or multiplicative forms $Q(s)=b f(s, s), b \geq 1$. 
It remains to establish that the sequences of optimal boundaries and value functions converge towards the corresponding ones of the original problem (2.1) as $n \rightarrow \infty$ and $z \rightarrow 0$. This property is established in our next theorem.

Theorem 4.1. Let Assumption 4.1 hold.

(a) Fix $z>0$. Then the limit $J(z, x, s):=\lim _{n \rightarrow \infty} J(z, n, x, s)$ exists finitely. Furthermore, $\lim _{z \rightarrow 0} J(z, x, s)=\sup _{\tau} \mathbb{E}_{(x, s)}\left\{\mathrm{e}^{-r \tau} f\left(X_{\tau}, S_{\tau}^{0}\right)\right\}$.

(b) Fix $s>0$. Then $\hat{a}_{s}$ approaches the optimal stopping boundary $a_{s}^{*}$ as $n \rightarrow \infty$ and $z \rightarrow 0$.

Proof. (a) Choose the terminal value function as $Q\left(s_{k}\right)=f\left(s_{k}, s_{k}\right)$. We see at once that this choice satisfies the conditions of Lemma 4.2 under Assumption 4.1. Moreover, with this choice, the value $J(z, n, x, s)$ constitutes an increasing sequence in $n$. To see this, fix $N \in \mathbb{N}$ and let $\left\{V_{k}^{N}\right\}_{k=0}^{N}$ be a sequence with respect to the number of steps $N$. Then $V_{N}^{N}=Q\left(s_{N-1}\right)=f\left(s_{N-1}, s_{N-1}\right)$. On the other hand, with the number of steps being $N+1$ we obtain $V_{N+1}^{N+1}=Q\left(s_{N}\right)=f\left(s_{N}, s_{N}\right)$, leading to

$$
\begin{aligned}
V_{N}^{N+1} & =\sup _{\tau} \mathbb{E}_{\left(s_{N-1}, s_{N}\right)}\left\{\mathrm{e}^{-r \tau} f\left(X_{\tau}, s_{N}\right) \mathbf{1}_{\left\{\tau<\gamma_{s_{N}}\right\}}+\mathrm{e}^{-r \gamma_{s_{N}}} Q\left(s_{N}\right) \mathbf{1}_{\left\{\tau \geq \gamma_{s_{N}}\right\}}\right\} \\
& \geq f\left(s_{N-1}, s_{N}\right) \\
& \geq f\left(s_{N-1}, s_{N-1}\right) \\
& =V_{N}^{N} .
\end{aligned}
$$

Consequently, $V_{k}^{N+1} \geq V_{k}^{N}$ for all $k \leq N$, so that especially $V_{0}^{N+1}=J(z, N+1, x, s) \geq$ $J(z, N, x, s)=V_{0}^{N}$.

Moreover, utilizing expression (4.2) and applying the assumed $s$-Hölder continuity, we can make the following estimation for an arbitrary $n \in \mathbb{N}$ :

$$
\begin{aligned}
J(z, n, x, s) & \leq \sup _{\tau} \mathbb{E}_{(x, s)}\left\{\mathrm{e}^{-r \tau} f\left(X_{\tau}, S_{\tau}^{0}+z\right) \mathbf{1}_{\left\{\tau<\gamma_{s_{n}}\right\}}+\mathrm{e}^{-r \gamma_{s_{n}}} Q\left(s_{n}\right) \mathbf{1}_{\left\{\tau \geq \gamma_{s_{n}}\right\}}\right\} \\
& \leq \sup _{\tau} \mathbb{E}_{(x, s)}\left\{\mathrm{e}^{-r \tau} f\left(X_{\tau}, S_{\tau}^{0}+z\right)\right\}+\mathbb{E}_{(x, s)}\left\{\mathrm{e}^{-r \gamma_{s_{n}}} Q\left(s_{n}\right)\right\} \\
& \leq \sup _{\tau}\left\{\mathbb{E}_{(x, s)}\left\{\mathrm{e}^{-r \tau} f\left(X_{\tau}, S_{\tau}^{0}\right)\right\}+\mathbb{E}_{(x, s)}\left\{\mathrm{e}^{-r \tau} M z^{\alpha}\right\}\right\}+\mathbb{E}_{(x, s)}\left\{\mathrm{e}^{-r \gamma_{s_{n}}} Q\left(s_{n}\right)\right\} \\
& \leq \sup _{\tau} \mathbb{E}_{(x, s)}\left\{\mathrm{e}^{-r \tau} f\left(X_{\tau}, S_{\tau}^{0}\right)\right\}+M z^{\alpha}+\mathbb{E}_{(x, s)}\left\{\mathrm{e}^{-r \gamma_{s_{n}}} Q\left(s_{n}\right)\right\} \\
& <\infty .
\end{aligned}
$$

The finiteness follows from Lemma 3.2. Since $J(z, n, x, s)$ is a bounded increasing sequence, it converges as $n \rightarrow \infty$. Since $\lim _{n \rightarrow \infty} \mathbb{E}_{(x, s)}\left\{\mathrm{e}^{-r \gamma_{s_{n}}} Q\left(s_{n}\right)\right\}=0$, we obtain

$$
J(z, x, s)=\lim _{n \rightarrow \infty} J(z, n, x, s) \leq \sup _{\tau} \mathbb{E}_{(x, s)}\left\{\mathrm{e}^{-r \tau} f\left(X_{\tau}, S_{\tau}^{0}\right)\right\}+M z^{\alpha} .
$$

On the other hand, utilizing again expression (4.2) we also obtain the inequality

$$
\begin{aligned}
J(z, x, s) & =\sup _{\tau} \mathbb{E}_{(x, s)}\left\{\mathrm{e}^{-r \tau} f\left(X_{\tau}, s+z \sum_{k=1}^{\infty} \mathbf{1}_{\left\{\gamma_{s_{k}}<\tau\right\}}\right)\right\} \\
& \geq \sup _{\tau} \mathbb{E}_{(x, s)}\left\{\mathrm{e}^{-r \tau} f\left(X_{\tau}, S_{\tau}^{0}-z\right)\right\} \\
& \geq \sup _{\tau} \mathbb{E}_{(x, s)}\left\{\mathrm{e}^{-r \tau} f\left(X_{\tau}, S_{\tau}^{0}\right)\right\}-M z^{\alpha} .
\end{aligned}
$$


Combining this with (4.3) we see that

$$
\sup _{\tau} \mathbb{E}_{(x, s)}\left\{\mathrm{e}^{-r \tau} f\left(X_{\tau}, S_{\tau}^{0}\right)\right\}-M z^{\alpha} \leq J(z, x, s) \leq \sup _{\tau} \mathbb{E}_{(x, s)}\left\{\mathrm{e}^{-r \tau} f\left(X_{\tau}, S_{\tau}^{0}\right)\right\}+M z^{\alpha},
$$

so that by letting $z \rightarrow 0$ we obtain $J(z, x, s) \rightarrow \sup _{\tau} \mathbb{E}_{(x, s)}\left\{\mathrm{e}^{-r \tau} f\left(X_{\tau}, S_{\tau}^{0}\right)\right\}$.

(b) The value function $V$ of the original problem (2.1) can be written as (cf. Theorem 3.2)

$$
V(x, s)=\sup _{\tau} \mathbb{E}_{(x, s)}\left\{\mathrm{e}^{-r \tau} f\left(X_{\tau}, s\right) \mathbf{1}_{\left\{\tau<\gamma_{s}\right\}}+\mathrm{e}^{-r \gamma_{s}} \sup _{\xi} \mathbb{E}_{(s, s)}\left\{\mathrm{e}^{-r \xi} f\left(X_{\xi}, S_{\xi}^{0}\right)\right\} \mathbf{1}_{\left\{\tau \geq \gamma_{s}\right\}}\right\},
$$

where $\tau$ and $\xi$ are admissible stopping times, and the supremum is attained with $\tau_{a_{s}^{*}}=\inf \{t \geq$ $\left.0 \mid X_{t} \leq a_{s}^{*}\right\}$, where $a_{s}^{*} \in\left(0, \tilde{x}_{s}\right)$ is the unique stopping boundary from Theorem 3.2.

On the other hand, the discretized problem can be written as

$$
\begin{aligned}
\lim _{z \rightarrow 0} J(z, x, s) & =\lim _{z \rightarrow 0} \sup _{\tau} \mathbb{E}_{(x, s)}\left\{\mathrm{e}^{-r \tau} f\left(X_{\tau}, s\right) \mathbf{1}_{\left\{\tau<\gamma_{s}\right\}}+\mathrm{e}^{-r \gamma_{s}} V_{1} \mathbf{1}_{\left\{\tau \geq \gamma_{s}\right\}}\right\} \\
& =\sup _{\tau} \mathbb{E}_{(x, s)}\left\{\mathrm{e}^{-r \tau} f\left(X_{\tau}, s\right) \mathbf{1}_{\left\{\tau<\gamma_{s}\right\}}+\mathrm{e}^{-r \gamma_{s}} \mathbf{1}_{\left\{\tau \geq \gamma_{s}\right\}} \lim _{z \rightarrow 0} V_{1}\right\},
\end{aligned}
$$

where the supremum is attained with $\tau_{\hat{a}_{s}}$, where $\hat{a}_{s} \in\left(0, \tilde{x}_{s}\right)$ is the unique stopping boundary. Now $J(z, s-, s)=V_{1}$ and according to part (a) $\lim _{z \rightarrow 0} J(z, s-, s)=\sup _{\tau} \mathbb{E}_{(s, s)}\left\{\mathrm{e}^{-r \tau}\right.$ $\left.f\left(X_{\tau}, S_{\tau}^{0}\right)\right\}$. Hence, we obtain the equality

$$
\begin{aligned}
\lim _{z \rightarrow 0} J(z, x, s)=\sup _{\tau} \mathbb{E}_{(x, s)}\left\{\mathrm{e}^{-r \tau} f\left(X_{\tau}, s\right) \mathbf{1}_{\left\{\tau<\gamma_{s}\right\}}\right. \\
\left.+\mathrm{e}^{-r \gamma_{s}} \sup _{\xi} \mathbb{E}_{(s, s)}\left\{\mathrm{e}^{-r \xi} f\left(X_{\xi}, S_{\xi}^{0}\right)\right\} \mathbf{1}_{\left\{\tau \geq \gamma_{s}\right\}}\right\},
\end{aligned}
$$

which coincides with (4.5). It follows that we have $\hat{a}_{s}=a_{s}^{*}$.

Theorem 4.1 demonstrates that the developed algorithm indeed converges to the proposed limit. However, it does not characterize the speed of convergence to the limit as the discretization step becomes smaller. This subject is addressed in the following result.

Corollary 4.1. Let Assumption 4.1 hold. Then the rate of convergence $\lim _{z \rightarrow 0} J(z, x, s)=$ $V(x, s)$ is of order $\mathcal{O}\left(z^{\alpha}\right)$.

Proof. From (4.4) we see straightforwardly that $J(z, x, s)=V(x, s)+\mathcal{O}\left(z^{\alpha}\right)$.

Unfortunately, Corollary 4.1 characterizes the convergence of the algorithm only in terms of the denseness of the applied discretization and not in terms of the number of steps. In order to characterize that, we would have to be able to estimate the difference $|V(x, s)-J(z, n, x, s)|$, which is a highly process-dependent quantity.

\subsection{A useful extension}

Let us present a discretization associated to the generalization introduced in Subsection 3.3. The proofs are analogous to those in Subsection 4.2, and are thus omitted.

Theorem 4.2. Let Assumption 3.1 hold. In addition, assume that

(a) $f(x, s)$ is s-Hölder continuous;

(b) $Q(s) \geq f(s, s)$ is such that $\lim _{t \rightarrow \infty} \mathbb{E}_{(x, s)}\left\{\mathrm{e}^{-r t} Q\left(S_{t}^{0}\right)\right\}=0$.

Then $\lim _{z \rightarrow \infty} \lim _{n \rightarrow \infty} J(z, n, x, s)=V(x, s)$, where $J$ is defined through (4.1). 
Proposition 4.1. Let the assumptions of Theorem 4.2 hold. In addition, assume that, for all $s>0$ and $Q$, there exists a unique stopping region $\mathfrak{S}_{s}^{Q}$ such that $\tau_{Q}^{*}=\left\{t \geq 0 \mid X_{t} \in \mathfrak{S}_{s}^{Q}\right\}$ provides the value for the auxiliary problem (3.1). Then

(a) $\lim _{z \rightarrow \infty} \lim _{n \rightarrow \infty} J(z, n, x, s)=V(x, s)$;

(b) $\lim _{z \rightarrow 0} \lim _{n \rightarrow \infty} \hat{\tau}_{\hat{\mathfrak{S}}_{s}}=\tau_{s}^{*}$, where $\hat{\tau}_{\hat{\mathfrak{S}}_{s}}=\inf \left\{t \geq 0 \mid X_{t} \in \hat{\mathfrak{S}}_{s}\right\}$ and $\hat{\mathfrak{S}}_{s}$ is the stopping region for the discretized problem with a state $s$, and $\tau_{s}^{*}$ is the optimal stopping time for the problem (2.1) with a state $s$.

Theorem 4.2 illustrates that, under the stated assumptions, the discretization approaches the value irrespective of whether the value is attained with a finite stopping time or not. In addition, if we know that, for all admissible $Q(s)$, the value of the auxiliary problem is attained with an admissible stopping time then the stopping region 'approaches' the stopping region of the initial problem as well. All in all, the generalization in Subsection 3.3 ensures that the proof of the existence of a solution to problem (2.1) reduces to the proof of the existence of a solution to the linear problem (3.1). Furthermore, the results above guarantee that these solutions can be attained numerically.

\section{Explicit illustration: perpetual lookback with floating strike}

In order to illustrate the algorithm developed in our paper, we now consider the valuation and optimal exercise of a perpetual lookback option with a floating strike. In this case the exercise payoff reads as $f(x, s)=(s-k x)$, where $k \in \mathbb{R}_{+}$is a known exogenously given constant. Therefore, our objective is to analyze and solve the stopping problem (cf. [9], [10], [19], [20], and [24])

$$
V(x, s)=\sup _{\tau} \mathbb{E}_{(x, s)}\left\{\mathrm{e}^{-r \tau}\left(S_{\tau}-k X_{\tau}\right)\right\}
$$

It is clear that, by letting $k \downarrow 0$, the problem becomes the valuation of a perpetual Russian option (cf. [27]). As our general findings indicate, in this case we have the following result.

Proposition 5.1. Assume that $\int_{0}^{\infty} \mathbb{E}_{(x, s)}\left\{\mathrm{e}^{-r t} S_{t}\right\} \mathrm{d} t<\infty$, that there is a single state $\tilde{x}_{s} \in \mathbb{R}_{+}$ so that $k(r x-\mu(x)) \lesseqgtr r s$ for $x \lesseqgtr \tilde{x}_{s}$, and that $\lim _{x \downarrow 0} \mu(x) \geq 0$. Then the value function of problem (5.1) reads as

$$
V_{a^{*}}(x, s)= \begin{cases}\frac{\left(s-k a_{s}^{*}\right) \psi^{\prime}\left(a_{s}^{*}\right)+k \psi\left(a_{s}^{*}\right)}{B L^{\prime}\left(a_{s}^{*}\right)} \varphi(x) & \\ +\frac{\left(k a_{s}^{*}-s\right) \varphi^{\prime}\left(a_{s}^{*}\right)-k \varphi\left(a_{s}^{*}\right)}{B L^{\prime}\left(a_{s}^{*}\right)} \psi(x) & \text { if } x \in\left(a_{s}^{*}, s\right), \\ s-k x & \text { if } x \in\left(0, a_{s}^{*}\right],\end{cases}
$$

where $a_{s}^{*}$ can be seen either as the limit boundary stated in Theorem 4.1 or, alternatively, as the solution of the ordinary differential equation

$$
a_{s}^{\prime}=\frac{\hat{\varphi}^{\prime}\left(a_{s}\right) \sigma^{2}\left(a_{s}\right)}{2 \hat{\varphi}\left(a_{s}\right)\left(r\left(k a_{s}-s\right)-k \mu\left(a_{s}\right)\right)},
$$

subject to the maximality principle. The optimal stopping time is $\tau^{*}=\inf \left\{t \geq 0 \mid X_{t} \leq a_{S_{t}}^{*}\right\}$.

\subsection{Geometric Brownian motion example}

Assume now that $X_{t}$ evolves according to a geometric Brownian motion characterized by the stochastic differential equation $\mathrm{d} X_{t}=\mu X_{t} \mathrm{~d} t+\sigma X_{t} \mathrm{~d} W_{t}$, where $\mu \in(-\infty, r)$ and $\sigma>0$. In this case, the decreasing and increasing fundamental solutions read as $\varphi(x)=x^{\gamma_{1}}$ and 
$\psi(x)=x^{\gamma_{2}}$, where

$$
\gamma_{i}=\frac{1}{\sigma^{2}}\left(\frac{1}{2} \sigma^{2}-\mu+(-1)^{i} \sqrt{\left(\frac{1}{2} \sigma^{2}-\mu\right)^{2}+2 \sigma^{2} r}\right)
$$

are the solutions of the characteristic equation $\frac{1}{2} \sigma^{2} \gamma(\gamma-1)+\mu \gamma-r=0$ for $i=1,2$. Note that $\gamma_{1}<0$ and, since $\mu<r$, we have $\gamma_{2}>1$. Under this setting, problem (5.1) can be solved explicitly (see [19] and [24]):

Proposition 5.2. When $X_{t}$ is a geometric Brownian motion, the value of the perpetual lookback (5.1) is

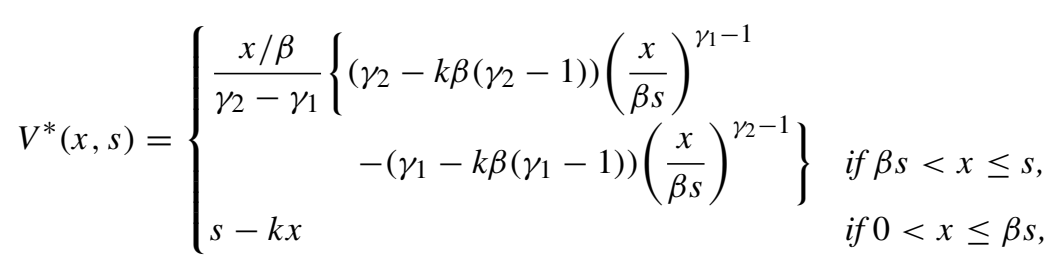

and the optimal stopping time is given by $\tau^{*}=\inf \left\{t \geq 0 \mid X_{t} \leq \beta S_{t}\right\}$, where $\beta$ is the unique solution to the equation

$$
\beta^{\gamma_{2}-\gamma_{1}}=\frac{\left(\gamma_{2}-1\right)\left(\gamma_{1}-k \beta\left(\gamma_{1}-1\right)\right)}{\left(\gamma_{1}-1\right)\left(\gamma_{2}-k \beta\left(\gamma_{2}-1\right)\right)} .
$$

The comparison between the exact and an approximate result are summarized in Table 1 . We see from the results in this table that $\hat{a}$ is decreasing while $\hat{V}$ is increasing in $n$ (as the proof of Theorem 4.1 indicates), and that the computing time is linear. Another positive feature is that the algorithm simultaneously produces approximations for the optimal boundary $a_{s}^{*}$ for other $s \mathrm{~s}$ as well along the discretized supremum process.

In Table 2 we see that while the original approximation for $a_{10}^{*}$ was very good, other estimates for $a_{15}^{*}, \ldots, a_{50}^{*}$ are also quite good, every single one being under half a percent away from the exact value.

TABLE 1: The values for the model are $(\sigma, \mu, r, k)=(0.2,0.05,0.08,1),(x, s)=(7,10)$, and, for the approximation, we chose $Q(s)=f(s, s) \equiv 0, z=0.1$. The exact values are $V(7,10)=4.03$ and $a_{10}^{*}=5.34$.

\begin{tabular}{rccc}
\hline$n$ & $V^{*}-\hat{V}$ & $\hat{a}-a^{*}$ & Time $(\mathrm{s})$ \\
\hline 100 & 0.89 & 1.0 & 0.2 \\
1000 & 0.27 & 0.21 & 3 \\
10000 & 0.049 & 0.036 & 29 \\
100000 & 0.0020 & 0.0014 & 288 \\
\hline
\end{tabular}

TABLE 2: A comparison of the exact values $a_{s}^{*}$ with the approximate values.

\begin{tabular}{crlc}
\hline$s$ & $a^{*}$ & $\hat{a}-a^{*}$ & $\left(\hat{a}-a^{*}\right) / a^{*}(\%)$ \\
\hline 15 & 8.0 & 0.0099 & 0.12 \\
20 & 10.7 & 0.021 & 0.19 \\
25 & 13.4 & 0.034 & 0.25 \\
30 & 16.0 & 0.048 & 0.30 \\
40 & 21.3 & 0.084 & 0.39 \\
50 & 26.7 & 0.13 & 0.47 \\
\hline
\end{tabular}




\subsection{Mean reverting diffusion}

To illustrate our findings in a somewhat more complicated setting, let $\mathrm{d} X_{t}=\mu X_{t}(\theta-$ $\left.X_{t}\right) \mathrm{d} t+\sigma X_{t} \mathrm{~d} W_{t}$, where $\mu, \theta, \sigma>0$ are exogenously given constants. The fundamental solutions are now $\psi(x)=x^{\gamma_{2}} M\left(\gamma_{2}, 1+\gamma_{2}-\gamma_{1}, 2 \mu \theta x / \sigma^{2}\right)$ and $\varphi(x)=x^{\gamma_{1}} U\left(\gamma_{1}, 1+\gamma_{1}-\right.$ $\left.\gamma_{2}, 2 \mu \theta x / \sigma^{2}\right)$, where $M: \mathbb{R}^{+} \rightarrow \mathbb{R}_{+}$and $U: \mathbb{R}_{+} \rightarrow \mathbb{R}_{+}$denote the confluent hypergeometric functions of the first and second kind, respectively (cf. [1, p. 504]), and $\gamma_{i}, i=1,2$, are as in (5.2). These functions are very difficult to handle analytically and, therefore, we numerically analyze the solution to (5.1) under the following parameter specifications: $\mu=0.05, \theta=0.1$, $\sigma=0.15, r=0.08$, and $k=1$.

Let us apply the algorithm. From Table 3 we see that choosing the highest possible state for $X_{t}$ to be $s_{n}=75$ or $s_{n}=200$ has only a minor impact on the solution. Thus, the choice $s_{n}=75$ is adequate for the estimation when $s \leq 10$. Moreover, since the $f(x, s)$ is now $s$-Lipschitz continuous with Lipschitz constant 1 , we see from Corollary 4.1 that we can quite surely say that $|J(x, s)-V(x, s)|<z$ for $s \leq 10$, where $J$ is our approximate function and $V$ the (unknown) optimal value function. In Table 4 we see the effect of changing the grid parameter $z$. The impact of increased volatility on the optimal boundary and the value are illustrated in Figure 2.

TABLE 3: The grid $z=0.1$ is fixed, and the differences $\left|J\left(0.1, n_{1}, 9.9,10\right)-J\left(0.1, n_{2}, 9.9,10\right)\right|$ are calculated, where $n_{i}$ is such that the highest state for $X_{t}$ is $s_{n_{i}}$.

\begin{tabular}{ccccc}
\hline \multirow{2}{*}{$s_{n}$} & \multicolumn{4}{c}{ Differences $\left|J\left(0.1, n_{1}, 9.9,10\right)-J\left(0.1, n_{2}, 9.9,10\right)\right|$} \\
\cline { 2 - 5 } & 50 & 75 & 100 & 200 \\
\hline 50 & - & $4.3 \times 10^{-5}$ & $4.3 \times 10^{-5}$ & $4.3 \times 10^{-5}$ \\
75 & & - & $6.6 \times 10^{-9}$ & $6.6 \times 10^{-9}$ \\
100 & & & - & $5.2 \times 10^{-13}$ \\
\hline Time (s) & 50 & 118 & 234 & 598 \\
\hline
\end{tabular}

TABLE 4: The initial point $(x, s)=(2,3)$ and the highest state $s_{n}=75$ is fixed. We compare how the solution changes as we change $z$ (in each case $n$ is chosen such that $s_{n}=75$ ).

\begin{tabular}{lccccc}
\hline \multicolumn{1}{c}{$z$} & $J(2,3)$ & $\hat{a}_{3}$ & $\hat{a}_{7}$ & $\hat{a}_{10}$ & Time $(\mathrm{s})$ \\
\hline 0.1 & 1.000889 & 1.97771 & 4.57640 & 6.44145 & 95 \\
0.01 & 1.000233 & 1.98858 & 4.58651 & 6.45046 & 958 \\
0.005 & 1.000209 & 1.98917 & 4.58707 & 6.45096 & 1875 \\
\hline
\end{tabular}
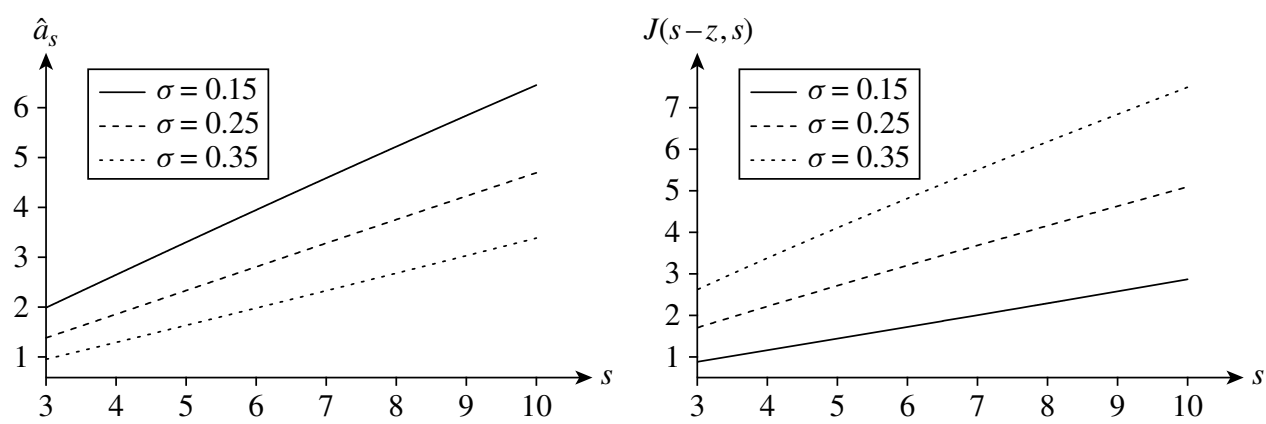

FIGURE 2: The stopping boundaries $\hat{a}_{s}$ and the values $J(s-z, s)$ are calculated for $s \in(3,10)$ and $\sigma=0.15,0.25,0.35$. We have chosen $z=0.01$ and $s_{n}$ to be 75 (with $\sigma=0.15$ ), 100 (with $\sigma=0.25$ ), and 150 (with $\sigma=0.35$ ). 


\section{Explicit illustration: $\pi$-option}

In order to utilize our findings on the generalized case introduced in Subsections 3.3 and 4.3, we will now consider the valuation and optimal exercise of a $\pi$-option introduced in [14]. In this case the exercise payoff reads as $f(x, s)=x^{\kappa} s^{\eta}-K$, where $\kappa, \eta, K \geq 0$ are known exogenously given constants. That is, we plan to analyze and solve the stopping problem

$$
V(x, s)=\sup _{\tau} \mathbb{E}_{(x, s)}\left\{\mathrm{e}^{-r \tau}\left(X_{\tau}^{\kappa} S_{\tau}^{\eta}-K\right)\right\} .
$$

Let $Q(s) \geq f(s, s)$ be a continuous function, and assume that $\lim _{t \rightarrow \infty} \mathbb{E}_{(x, s)}\left\{\mathrm{e}^{-r t} Q\left(S_{t}\right)\right\}=$ 0 . Consider the auxiliary problem

$$
V^{Q}(x, s)=\sup _{\tau} \mathbb{E}_{x}\left\{\mathrm{e}^{-r \tau}\left(X_{\tau}^{\kappa} S_{\tau}^{\eta}-K\right) \mathbf{1}_{\left\{\tau<\gamma_{s}\right\}}+\mathrm{e}^{-r \gamma_{s}} Q(s) \mathbf{1}_{\left\{\tau \geq \gamma_{s}\right\}}\right\} .
$$

Applying our tools, we obtain the following result (cf. Section 4 of [14]).

Proposition 6.1. Assume that $\int_{0}^{\infty} \mathbb{E}_{x}\left\{\mathrm{e}^{-r t} f\left(S_{t}, S_{t}\right)\right\} \mathrm{d} t \leq \infty$ and that, for each $s>0$, there exists $\tilde{x}_{s} \in(0, s]$ such that $(\mathcal{A}-r) f(x, s) \gtreqless 0$ for all $x \lesseqgtr \tilde{x}_{s}$. Then, for each $s>0$, the value for (6.1) is finite and the optimal stopping time is $\tau_{\mathfrak{S}_{s}}=\inf \left\{t \geq 0 \mid X_{t} \in \mathfrak{S}_{s}\right\}$, where $\mathfrak{S}_{s}$ is $s$-dependent and is either $\varnothing$ or of the form $\left[u_{s}^{*}, y_{s}^{*}\right]$, where $0<u_{s}^{*} \leq y_{s}^{*}<s$ are uniquely determined.

Proof. Let us apply Proposition 3.1 and Corollary 3.1, and let us show that, for each $s>0$ and $Q(s)$, the stopping region $\mathfrak{S}_{s}^{Q}$ of the auxiliary problem (6.2) is of the claimed form. Denote by $C_{s}^{Q}$ the continuation region at a fixed state $s>0$. Clearly, $f(0, s)<0$ for all $s>0$, which implies that the region near the boundary 0 belongs to the continuation region. It follows from Corollary 4 of [21] (see also Theorem 2 of [6]) that $\left(0, \min \left\{u_{s}^{*}, s\right\}\right)$ belongs to a continuation region, where $u_{s}^{*}=\operatorname{argmax}_{x \in \mathbb{R}_{+}}\{f(x, s) / \psi(x)\}$. Moreover, under our assumptions, $u_{s}^{*}$ is unique (cf. Lemma 3.6 of [3]). If $u_{s}^{*}>s$ then $(0, s) \subset C^{Q}$ and $\mathfrak{S}_{s}^{Q}=\varnothing$. Assume now that $s$ is such that $u_{s}^{*}<s$. We know by Dynkin's formula that $u_{s}^{*}>\tilde{x}_{s}$. Now, proceeding as in the proof of Lemma 3.1, we see that there exists a unique $y_{s}^{*} \in\left[u_{s}^{*}, s\right)$ maximizing $v(y, x, s)$ (see (3.3)) for all $x \in\left(y_{s}^{*}, s\right)$. Moreover, either the derivative $v_{y}^{\prime}\left(y_{s}^{*}, x, s\right)=0$ and $y_{s}^{*} \geq u_{s}^{*}$ or $v_{y}^{\prime}\left(y_{s}^{*}, x, s\right)<0$ and $y_{s}^{*}=u_{s}^{*}$. In the former case $\mathfrak{S}_{s}^{Q}=\left[u_{s}^{*}, y_{s}^{*}\right]$ and in the latter case $\mathfrak{S}_{s}^{Q}=\varnothing$. The optimality of the stopping time $\tau_{\mathfrak{S}_{s}^{Q}}$ for the auxiliary problem follows after noting that the resulting value is an $r$-excessive majorant of the exercise payoff. The results now follow from Proposition 3.1 and Corollary 3.1.

The stopping region $\mathfrak{S}_{s}$ and its dependence on $s$ can be characterized more closely under more restricting assumptions. However, since our purpose is not to provide an exhaustive treatment of this subject, we will not go deeper into the analysis of the $\pi$-option.

\subsection{Numerical example}

By Theorem 4.2, our discretization works for the $\pi$-option. In our numerical illustration we have chosen $\kappa=0.9, \eta=1, K=9$, and $Q(s)=f(s, s)$. Although the numerics indicate that the algorithm also converges for $\eta>1$, we were not able to prove the convergence in Theorem 4.1 without Hölder continuity.

6.1.1. Geometric Brownian motion. Let the setting be as in Subsection 5.1. In [14] the valuation of the $\pi$-option was solved under the geometric Brownian motion, which gives us a baseline for our numerical approximations. 
TABLE 5: The value of $\pi$-option for geometric Brownian motion. The values for the model are $(\sigma, \mu, r)=$ $(0.2,0.03,0.1),(x, s)=(10,13)$, and $z=0.25$. The exact values are: $V(10,13)=115.4$ and $y_{13}^{*}=$ 7.076.

\begin{tabular}{rllc}
\hline \multicolumn{1}{c}{$n$} & $V^{*}-\hat{V}$ & $\hat{y}-y_{13}^{*}$ & Time $(\mathrm{s})$ \\
\hline 400 & 7.50 & 0.6 & 0.7 \\
4000 & 2.5 & 0.18 & 4.7 \\
40000 & 0.70 & 0.050 & 47 \\
400000 & 0.07 & 0.0053 & 467 \\
\hline
\end{tabular}

TABLE 6: The value of the $\pi$-option in the case of a mean reverting diffusion. The values for the model are $(\sigma, \gamma, \mu, r)=(0.2,0.1,0.03,0.08)$ and $(x, s)=(11,13)$. The highest state $s_{n}=75$ is fixed. We compare how the solution changes as we change $z$ (in each case $n$ is chosen such that $s_{n}=75$ ). Now $u_{13}^{*}=1.40$.

\begin{tabular}{llrccc}
\hline \multicolumn{1}{c}{$z$} & $\hat{V}(11,13)$ & \multicolumn{1}{c}{$\hat{y}_{13}$} & $\hat{y}_{15}$ & $\hat{y}_{20}$ & Time (s) \\
\hline 0.1 & 105.470 & 9.9854 & 11.8577 & 16.5847 & 38 \\
0.01 & 105.342 & 10.0184 & 11.8919 & 16.6212 & 300 \\
0.005 & 105.335 & 10.0202 & 11.8938 & 16.6233 & 600 \\
0.0025 & 105.332 & 10.0211 & 11.8947 & 16.6243 & 1200 \\
\hline
\end{tabular}

From Table 5 we see that in about 50 seconds we were able to attain results that are within a $1 \%$ error margin. Note that $u_{13}^{*}:=\operatorname{argmax}\{f(x, 13) / \psi(x)\}=1.29$ is independent of $Q(s)$, $z$, and $n$, and is always exact.

6.1.2. Mean reverting diffusion. Let the setting be as in Subsection 5.2. Now there is no known exact solution. As was the case earlier (Subsection 5.2), it has only a minor impact on the solution whether we choose the highest possible state for $X_{t}$ to be $s_{n}=70$ or $s_{n}=200$. Therefore, the choice $s_{n}=75$ is adequate for our estimation. The results are summarized in Table 6.

\section{Acknowledgements}

The authors are grateful to an anonymous referee for constructive comments and suggested improvements, and additions to the contents of our paper. The authors would also like to thank Prof. Paavo Salminen for pointing out a mistake in the original version of Lemma 3.2. Luis H. R. Alvarez E. gratefully acknowledges financial support from the OP Bank Group Research Foundation.

\section{References}

[1] Abramowitz, M. and Stegun, I. A. (1964). Handbook of Mathematical Functions with Formulas, Graphs, and Mathematical Tables (National Bureau Standards App. Math. Ser. 55). U.S. Government Printing Office, Washington, D.C.

[2] Alvarez, L. H. R. (2003). On the properties of $r$-excessive mappings for a class of diffusions. Ann. Appl. Prob. 13, 1517-1533.

[3] Alvarez, L. H. R. AND Lempa, J. (2008). On the optimal stochastic impulse control of linear diffusions. SIAM J. Control Optimization 47, 703-732.

[4] BabBs, S. (2000). Binomial valuation of lookback options. J. Econom. Dynam. Control 24, 1499-1525.

[5] Beibel, M. ANd Lerche, H. R. (1997). A new look at optimal stopping problems related to mathematical finance. Statistica Sinica 7, 93-108. 
[6] Beibel, M. and Lerche, H. R. (2000). A note on optimal stopping of regular diffusions under random discounting. Teor. Veroyat. Primenen. 45, 657-669 (in Russian).

[7] Borodin, A. N. And Salminen, P. (2002). Handbook of Brownian Motion-Facts and Formulae, 2nd edn. Birkhäuser, Basel.

[8] Christensen, S., Salminen, P. and TA, B. Q. (2013). Optimal stopping of strong Markov processes. Stoch. Process. Appl. 123, 1138-1159.

[9] Conze, A. And Viswanathan, R. (1991). Path dependent options: the case of lookback optionsJ. Finance 46, 1893-1907.

[10] Douady, R., Shiryaev, A. N. And Yor, M. (2000). On probability characteristics of "downfalls" in a standard Brownian motion. Theory Prob. Appl. 44, 29-38.

[11] Föllmer, H. ANd KNispel, T. (2007). Potentials of a Markov process are expected suprema. ESAIM Prob. Statist. 11, 89-101.

[12] Glover, K., Hulley, H. And Peskir, G. (2013). Three-dimensional Brownian motion and the golden ratio rule. Ann. Appl. Prob. 23, 895-922.

[13] Graversen, S. E. AND PeŠKIR, G. (1998). Optimal stopping and maximal inequalities for linear diffusions. $J$. Theoret. Prob. 11, 259-277.

[14] Guo, X. and Zervos, M. (2010). $\pi$ options. Stoch. Process. Appl. 120, 1033-1059.

[15] Hobson, D. (2007). Optimal stopping of the maximum process: a converse to the results of Peskir. Stochastics 79, 85-102.

[16] JiAnG, L. AND DAI, M. (2004). Convergence of binomial tree methods for European/American path-dependent options. SIAM J. Numer. Anal. 42, 1094-1109.

[17] Karatzas, I. And Shreve, S. E. (1988). Brownian Motion and Stochastic Calculus. Springer, New York.

[18] Kyprianou, A. And OtT, C. (2014). A capped optimal stopping problem for the maximum process. Acta Appl. Math. 129, 147-174.

[19] Lerche, H. R. And Urusov, M. (2007). Optimal stopping via measure transformation: the Beibel-Lerche approach. Stochastics 79, 275-291.

[20] Magdon-Ismail, M., Atiya, A. F., Pratap, A. and Abu-Mostafa, Y. S. (2004). On the maximum drawdown of a Brownian motion. J. Appl. Prob. 41, 147-161.

[21] Матомӓкі, P. (2013). Optimal stopping and control near boundaries. Preprint. Available at http://uk.arxiv.org/ abs/1308.2478.

[22] OвŁó,, J. (2007). The Maximality Principle Revisited: On Certain Optimal Stopping Problems (Lecture Notes Math. 1899). Springer, Berlin, pp. 309-328.

[23] Отт, C. (2013). Optimal stopping problems for the maximum process. Doctoral Thesis. University of Bath.

[24] Pedersen, J. L. (2000). Discounted optimal stopping problems for the maximum process. J. Appl. Prob. 37, 972-983.

[25] Peskir, G. (1998). Optimal stopping of the maximum process: the maximality principle. Ann. Prob. 26, 1614-1640.

[26] Peskir, G. And Shiryaev, A. (2006). Optimal Stopping and Free-boundary Problems. Birkhäuser, Basel.

[27] Shepr, L. And Shiryaev, A. N. (1993). The Russian option: reduced regret. Ann. Appl. Prob. 3, 631-640. 